\title{
Myristoylated Alanine-Rich protein Kinase C Substrate (MARCKS) expression modulates the metastatic phenotype in human and murine colon carcinoma in vitro and in vivo
}

\author{
Krista Rombouts a,c,* Vinicio Carloni ${ }^{\text {a,c }}$, Tommaso Mello ${ }^{b, c}$, Sara Omenetti ${ }^{\text {a,c }}$, Sara Galastri ${ }^{\text {a,c }}$, \\ Stefania Madiai ${ }^{\text {a,c }}$, Andrea Galli ${ }^{\text {b,c }}$, Massimo Pinzani ${ }^{\text {a,c,d }}$ \\ a Department of Internal Medicine, University of Florence, Largo Brambilla 3, 50134 Florence, Italy \\ ${ }^{\mathrm{b}}$ Department of Clinical Pathophysiology, Viale Pieraccini 6, 50139 Florence, Italy

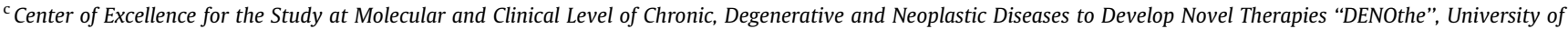 \\ Florence, Florence, Italy \\ ${ }^{\mathrm{d}}$ Royal Free Campus, Division of Medicine, UCL Institute for Liver and Digestive Health, Rowland Hill Street, London NW3 2PF, London, United Kingdom
}

\section{A R T I C L E I N F O}

\section{Article history:}

Received 17 December 2012

Accepted 23 January 2013

\section{Keywords:}

MARCKS

Tumour stromal cells

Actin reorganization

Aurora B kinase/AIM-1

Invasion/migration

Syngeneic-orthotopic murine model

\begin{abstract}
A B S T R A C T
Several actin-binding proteins have been shown to be altered in metastatic cell lines and tumours and, in particular, Myristoylated Alanine-Rich protein Kinase C substrate (MARCKS) has been implicated in the pathogenesis of various highly metastatic epithelial malignancies. Considering that a large percentage of deaths due to colorectal cancer (CRC) are consequent to hepatic metastasization, aim of this study was to elucidate the involvement and mechanism of MARCKS in CRC by employing in vitro and in vivo approaches. Loss-of and-gain-on function approaches of MARCKS were employed in two human CRC cell lines: Clone A cells expressing MARCKS and LoVo cells known to have a frameshift mutation of MARCKS i.e. typically for MSI-H CRC. The data unveiled that altering MARCKS expression suppresses cell motility and invasion in human colon carcinoma cells when conditioned medium of liver-specific stromal cells (hepatic stellate cells) was used as chemoattractant. Depletion or re-expression of MARCKS inhibited proliferation with a reduction in expression of the mitotic regulator Aurora $B$ kinase (AURKB), whereas AURKB-depletion did not modify MARCKS expression. In murine colon carcinoma CT26 cells, shRNA MARCKS-depletion reduced motility and invasion, and induced an aberrant, prolonged mitotic process. Significantly less metastases were produced in a syngeneic model of colon metastasis by MARCKSdepleted CT26 in comparison to CT26-tumour challenged mice. In conclusion, MARCKS plays an articulated role in the progression of colorectal cancer and might represent a suitable target to interfere and overcome the invasive behaviour of colon carcinoma cells at primary and distant sites.
\end{abstract}

(c) 2013 Elsevier Ireland Ltd. All rights reserved.

\section{Introduction}

Tumour growth and progression is not entirely due to genetic aberrations but also reflective of tumour cell plasticity. To metastasize, tumour cells must achieve a higher cell membrane plasticity and motion which are largely driven by the actin cytoskeleton and relies on the actin-based cytoskeleton reorganization [1]. Actin is the major constituent of the microfilament system and shows a high degree of structural plasticity. This plasticity is provided by the interplay between the monomeric, oligomeric and polymeric forms of actin and specialized actin-binding proteins that control the polymerisation and de-polymerisation of actin [2]. Several of

\footnotetext{
* Corresponding author at: Department of Internal Medicine, University of Florence, Largo Brambilla 3, 50134 Florence, Italy. Tel.: +39 0554271084; fax: +39 0554271065.

E-mail address: k.rombouts@dmi.unifi.it (K. Rombouts).
}

these actin-binding proteins are up- or down-regulated in metastatic cell lines and tumours. In particular, recent studies have identified Myristoylated Alanine-Rich C Kinase Substrate (MARCKS) to be implicated in the pathogenesis of various malignancies such as pituitary and thyroid cancer [3], breast cancer [4], melanoma [5], glioblastoma multiforme cancer [6,7], and cholangiocarcinoma [8].

Overall, the function of MARCKS in these malignancies seems to be multifaceted and depends on cell type and tumour origin. The identification of MARCKS as a frequent target of mutation in colorectal carcinomas and small intestinal adenocarcinomas with MSI$\mathrm{H}$ [9-11] certainly adds more complexity to the involvement of MARCKS in tumour formation. Indeed, the observed mutation in the effector domain of MARCKS, which contains the actin bindings domains, $[12,13]$ may lead to an aberrant re-organization of the actin cytoskeleton affecting processes such as motility, invasion and proliferation. A defect in the actin cytoskeleton architecture could 
then be associated with an aberrant cell cycle. Indeed, an aberrant regulation or localization of the mitotic regulator AURKB has been linked to genomic instability, tumourigenesis, tumour progression, and poor prognosis in a wide variety of cancer types including CRC [14-16]. Along these lines, we have previously demonstrated a close interaction between MARCKS and AURKB during mitosis. In particular, the specific cellular distribution of AURKB during the different mitotic steps depends on the presence of MARCKS and MARCKS regulation of actin filament formation [17].

Overall, the functional role of MARCKS during colorectal tumour progression and metastasization is largely unknown. Therefore, the aim of the present study was to investigate the functional role of MARCKS in vitro, by performing gain-on and-of experiments in CRC cell lines and in vivo by using a syngeneic murine model of liver metastasis.

\section{Materials and methods}

\subsection{Reagents}

Antibodies against alpha-smooth muscle actin ( $\alpha$-SMA) (mouse ascites, clone 1A4), $\beta$-actin (AC-15) and anti-actin (rabbit, 20-33) and all reagents used in this study are from Sigma Aldrich (Saint Louis, MI) or otherwise mentioned. Antibodies against AURKB (AIM-1) were purchased from Sigma (rabbit, A5102) or BD Transduction Laboratories (mouse, AIM-1). Primary antibodies against MARCKS ( $\mathrm{N}-19$ and M20), P-MARCKS (Ser159/163), $\alpha$-tubulin (H-300) were purchased from Santa Cruz Biotechnology as well as their secondary antibodies, respectively HRP-conjugated goat or rabbit IgG. All antibodies were used according to the manufacturer's manual.

\subsection{Cells and cell culture}

All cell lines were obtained from American Type Culture Collection (Manassas, VA) and were cultured in RPMI completed with $2 \mathrm{mM} / \mathrm{L}$ glutamine, $1.0 \mathrm{mM} / \mathrm{L}$ sodium pyruvate and supplemented with $10 \%$ Foetal Bovine Serum (FBS) (all provided by GIBCO Laboratories, NY). Human hepatic stellate cells (hHSCs) were isolated from wedge sections of normal human liver unsuitable for transplantation, as extensively described elsewhere [18]. Cells were cultured in Iscove's Modified DMEM supplemented with $0.6 \mathrm{U} / \mathrm{ml}$ insulin, $2 \mathrm{mM} / \mathrm{L}$ glutamine, $0.1 \mathrm{mM} / \mathrm{L}$ nonessential amino acids, $1.0 \mathrm{mM} / \mathrm{L}$ sodium pyruvate and $20 \% \mathrm{FBS}$. Harvesting the conditioned medium of hHSC ( $\mathrm{hHSC}-\mathrm{CM}$ ) was performed at passage 5 . At these stage, cells show functional and ultrastructural features of fully activated HSC [19]. Mice (mHSC) were isolated from liver Balb/c mice followed by density gradient centrifugation and culturing conditions as mentioned before. Conditioned medium of activated hHSC-CM and mHSC (mHSC-CM) was harvested after $48 \mathrm{~h}$ of incubation in serum-free medium. All cell lines were cultured under standard conditions in a humidified incubator under $5 \% \mathrm{CO}_{2}$ in air at $37^{\circ} \mathrm{C}$.

Conditioned medium from human or murine HSC was employed as a specific stimulus for CRC cell migration and invasion assays. This choice was based on previous published evidence indicating that activated HSC produce and secrete soluble factors and chemokines that promote a pro-inflammatory microenvironment, tissue angiogenesis and tumour progression. Therefore, activated hHSC favour the "homing" of tumour cells in the liver microenvironment [20-22]. Hence, the conditioned medium of activated hHSC is as an excellent and specific tool to investigate the possible cross-talk between cancer cells and hHSC during chemotaxis.

\subsection{Protein extraction, immunoprecipitation and Western blot analysis}

For immunoblot analysis whole cell lysates were separated on SDS-PAGE, transferred to nitrocellulose, and immunoblotted as described previously [23]. An antibody against $\alpha$-SMA, total actin, $\alpha$-tubulin or PCNA was used to demonstrate equal loading.

\subsection{RNA interference and plasmid transfection}

Transient gene silencing: in Clone A cells was performed by using human MARCKS validated SMARTpool siRNA (M-004772-00). RNAi against human AURKB in Clone A cells and LoVo cells was performed by using validated SMARTpool siRNA (M-003326-02) (Custom SMARTpool siRNA Design Service of Dharmacon, Lafayette, CO). For transient transfection, siLentFect ${ }^{\mathrm{TM}}$ Lipid Reagent (BioRad Laboratories, CA) was employed. Briefly, $3 \times 10^{5}$ cells were cultured per 6 -well plate. After $24 \mathrm{~h}$
A



B



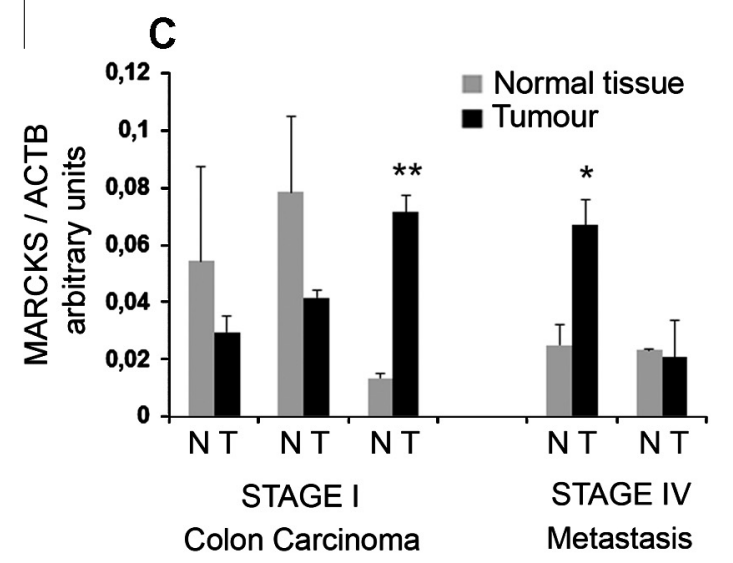

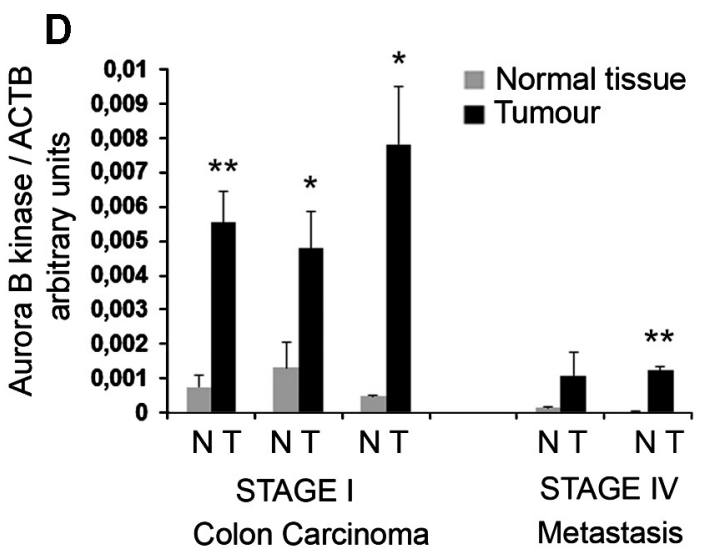



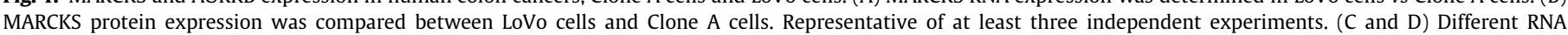

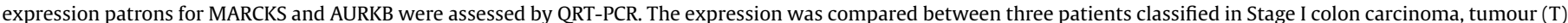

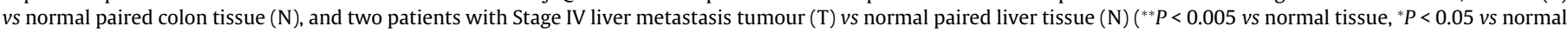
tissue). 
$30 \mathrm{nM}$ AURKB siRNA or $50 \mathrm{nM}$ MARCKS SiRNA was mixed with $5 \mu \mathrm{l}$ of siLentFect ${ }^{\mathrm{TM}}$ and added to the cells. Scrambled control siRNA was used in every transfection experiment [23].

Stable gene silencing: Murine colon carcinoma CT26 cells were stable transfected with murine validated short Hairpin RNA (TR512267, 4 shRNA constructs with puromycin cassette, containing two control shRNA) (OriGene Technologies, MD). Briefly, $3 \times 10^{5}$ cells were cultured in a 6 -well plate and after 24 h cells were transfected with $1 \mu \mathrm{g} / 3 \mathrm{ml}$ of shRNA construct using the Mirus 2300 Trans-IT-LT1 transfection reagent (Mirus Bio LLC, WI) and $8 \mu \mathrm{l} / \mathrm{ml}$ of puromycin was added to the medium. Single cell colonies were selected and experiments were performed on single cell colonies of the 4 th generation.

MARCKS plasmid transient transfection assay: LoVo cells were transiently transfected with a human MARCKS plasmid (WT-PJ7) or empty vector using the LipofectAMINE reagent (Life Technologies, Invitrogen). Furthermore, two different MARCKS cDNA mutations were investigated: (1) a myristoylated mutant (PJ7-G1A, Myr-m) where the $\mathrm{N}$-terminal glycine was replaced by an alanine that is unable to insert MARCKS protein into the plasma membrane but still contains the functional domain, and (2) a deletion mutant of the PSD (PJ7-PSD, PSD-m) which is replaced by Proline-Glycine. All plasmids were kindly given by Dr. S. Manenti (INSERM, Toulouse Cedex, France) $[5,24]$. Functional experiments were performed $48 \mathrm{~h}$ after each transfection and the transfection efficiency was evaluated by employing proteinand RNA analysis.

\subsection{Boyden chamber assays}

Briefly, Boyden chambers were equipped with 8 - $\mu$ m pore filters Poretics (Livermore, CA) and coated with rattail collagen (Collaborative Biomedical Products, MA) or Matrigel as previously described [20,23]. After employing gain-on or gain-of studies against MARCKS, cells were trypsinized and re-suspended in serum-free medium at a concentration of $6 \times 10^{4}$ cells/200 $\mu \mathrm{l}$ and added to the upper Boyden chamber. The conditioned medium of human HSC (hHSC-CM) or mouse HSC (mHSC-CM) was used as chemoattractant depending on the cell line species under investigation. After overnight incubation, the cells migrating to the underside of the filters were fixed, stained with Giemsa, mounted and viewed at $40 \times$ magnification. Data are the average of cell counts obtained in 10 randomly chosen high-power fields (HPF).

\subsection{RNA isolation and $Q R T-P C R$}

Total RNA was isolated with NucleoSpin RNAII (Macherey Nagel) according to the manufacturer's manual and $0.4 \mu \mathrm{g}$ aliquots were reverse-transcribed with random primers using a Reverse Aid M-MuLV reverse transcription kit (Fermentas). Target cDNA levels were quantified by SYBRE green-based RT-PCR using specific Taqman gene expression-assays (Applied Biosystems) with supplied probes on a Rotor Gene 6000 (Corbett Research).

Expression data from three male patients with colon rectal cancer and paired tissue classified in Stage I (T2, N0, MX), and two patients classified in Stage IV (TX, NX, M1) was generated from total RNAs obtained from OriGene Technologies (CR561166-CR561168, CR56277-CR562676, CR562687-CR562700, CR559462CR563007, CR560798-CR560801). Additional sample information and pathology reports are available on Origene Technologies website. Measured mRNA levels were normalized to those of $\beta$-actin or GAPDH as internal control and performed in triplicate. The relative levels of RNA were determined using the efficiency-corrected threshold cycle $(\Delta \mathrm{CT})$ method.

\subsection{In vivo liver metastasis assay}

Experimental protocols were conducted according to established international guidelines and with the approval of the National Regulatory Authorities and institutional guidelines. Six week old BALB/c mice (20 g, Charles River Laboratories, Italy) were tumour-challenged employing an intrasplenic bolus injection of scrambled control CT26 cells or MARCKS shRNA-depleted CT26 cells $\left(5 \times 10^{4} / 50 \mu \mathrm{l}\right.$ PBS1X). Sham-operated mice were obtained by performing the same surgical operation and injected with vehicle. Each group contained five animals. As was empirically proven, all CT26-tumour challenged mice developed liver metastasis. Mice were sacrificed after 23 days. Liver tissue was taken, snap-frozen or fixed in $10 \%$ buffered formaldehyde and embedded overnight in paraffin. This was followed by a standard haematoxylin-eosin staining as described previously [17].

\subsection{Cell proliferation assays}

MTT assay: scrambled control cells and shRNA MARCKS-depleted CT26 cells were seeded into a 12-well culture plate at $4 \times 10^{4}$ cells per well, performed in 6 -fold for each condition. Proliferation was measured by employing the MTT assay with key component 3-(4,5-dimethylthiazol-2-yl)-2,5-diphenyltetra zolium bromide (MTT) according to the manufacturer's manual (Sigma Aldrich).

DNA synthesis: DNA synthesis was measured as the amount of [3H] TdR incorporated into trichloroacetic acid-precipitable material, as described elsewhere [25]. Methyl-[3H]-thymidine was from New England Nuclear (Milan, Italy).
Cell count: after $48 \mathrm{~h}$ of transient transfection cells were trypsinized, stained with Trypan blue and counted.

\subsection{Video time lapse imaging}

To determine the impact of MARCKS expression on cell cycle, time lapse microscopy was employed that enables tracking of cell cycle progression at the single cell level [17] Video microscopy was performed by culturing cells in a micro-incubator (Pecon) with controlled humidity, temperature and $\mathrm{CO}_{2}$, mounted on inverted Leica AM6000 microscope with fully motorized stage. ShRNA MARCKS-depleted CT26 cells and scrambled control cells were followed over $24 \mathrm{~h}$. Experiments were performed acquiring multiple fields of view for each experimental condition by using oblique illumination and a 20X air objective. Multi-field acquisition was done using Leica LasAF software. Image analysis was performed by Image J software and Adobe PhotoShop.

Duration time of mitosis (DTM): mitotic entry was scored by the first image showing extensive cell rounding and is defined as the time (start $t 0$ ), whereas mitotic exit was scored as the last image showing the pinching-off of the two daughter cells $[17,26-28]$. The duration time of mitosis was measured for each mitotic cell in both conditions thus calculating the total sum of the DTM and the average DTM. Error bars represent standard error. The obtained average DTM of scrambled control cells (marked as $t$ ) was used as a cut-off value and compared with the DTM in MARCKS-depleted cells (marked as $>t$ ).

\subsection{Statistical analysis}

The statistical differences were determined by unpaired two-tailed Student's $t$ test. Two groups were considered statistically significant when $P$ value was less than 0.05 or 0.005 as mentioned in the figure legends.
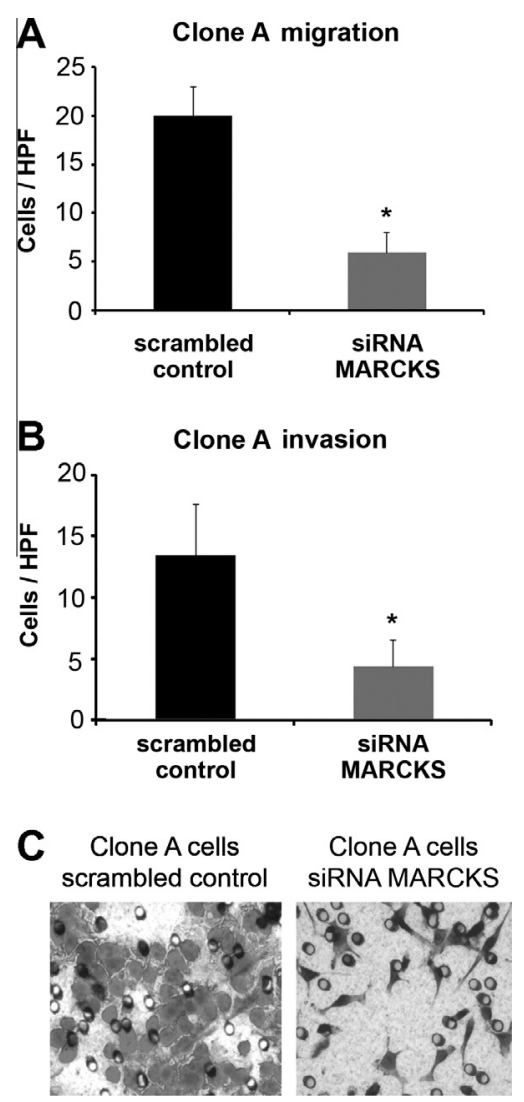

Fig. 2. RNAi of MARCKS in Clone A cells inhibits cell migration and invasion. (A) Boyden chamber chemotaxis assay was performed using as chemoattractant the conditioned medium (CM) of human hepatic stellate cells (hHSCs-CM). Values are expressed as cells migrated per High Power Field (HPF) and are shown as mean \pm SD of four independent experiments. ${ }^{*} P<0.05$, vs scrambled control cells towards hHSC-CM. (B) Invasion Matrigel assay was performed using hHSC-CM as chemoattractant. Values are shown as mean $\pm S D$ of four independent experiments. ${ }^{*} P<0.05$, vs scrambled control cells towards hHSC-CM. (C) The reduced chemotaxis of MARCKS-depleted Clone A cells towards hHSC-CM coincides with morphological changes. 


\section{Results}

3.1. The expression profile of MARCKS and AURKB in human colon carcinoma cell lines and in surgical specimens

In a first set of experiments, mRNA and protein expression of MARCKS were investigated in two different human colorectal carcinoma cell lines by employing QRT-PCR and Western blot, respectively. Clone A cells were originally isolated from a poorly differentiated human colon adenocarcinoma, whereas LoVo cells are defined as a DNA mismatch repair (MMR) deficient cell line, expressing a frameshift mutation for MARCKS inducing a truncated protein $[9,10]$.

MARCKS mRNA expression in LoVo cells was detectable but at an extreme low level ( $<0.001$ arbitrary units) when compared to MARCKS mRNA expression in Clone A cells (Fig. 1A). Analysing the protein expression level of MARCKS in both cell lines revealed that LoVo cells do not express MARCKS protein, whereas MARCKS was abundantly present in Clone A cells (Fig. 1B).

These in vitro findings suggest that MARCKS expression could be characterized by high variability in different colorectal carcinomas. This was also suggested by evidence obtained by investigating mRNA expression of MARCKS and AURKB in surgical samples of human colon carcinoma. A total of five paired samples from individual cases of CRC were investigated. Three of these samples were from patients classified in Stage I (T2, N0, MX) while two samples were from patients classified in Stage IV (TX, NX, M1) based on their case ID reported by OriGene Technologies. As shown in Fig. 1C, the mRNA expression level of MARCKS was strongly upregulated in tumour vs normal tissue in one sample classified Stage I while no difference was observed in the resulting two samples.
MARCKS expression showed to be up-regulated in one sample classified in Stage IV tumour vs normal liver tissue, whereas MARCKS expression level did not varied between tumour and normal tissue in one sample. Aurora kinase B mRNA expression was up-regulated in tumour $v s$ normal tissue in all Stage I samples (Fig. 1D). In two samples classified in Stage IV, AURKB expression showed to significantly be up-regulated only in one out of two cases.

\subsection{Loss of function of MARCKS interferes with cell motility and invasion in Clone A cells}

In this group of experiments we investigated whether changing MARCKS protein expression, and therefore the actin cytoskeleton, could affect cell migration and invasion of Clone A cells when conditioned medium of activated hHSC (hHSC-CM) was used as chemoattractant [20-22]. Therefore, RNAi against MARCKS was performed, followed by modified Boyden chamber migration-and invasion assays. As shown in Fig. 2A, cell motility of scrambled control cells was induced by hHSC-CM, whereas migration of MARCKS-depleted cells was significantly reduced.

A similar pattern of invasion was obtained when MARCKSdepleted Clone A cells were challenged to invade a modified Boyden chamber coated with Matrigel. Invasion of scrambled control cells was induced by hHSC-CM, whereas it was strongly inhibited in MARCKS-depleted cells (Fig. 2B).

The observed inhibition in chemotaxis towards hHSC-CM, known to be a strong hepatic chemoattractant [20-22], coincides with evident morphological changes. Scrambled control cells showed a more epithelial phenotype, whereas MARCKS-depleted Clone A cells were characterized by mesenchymal-like features (Fig. 2C).
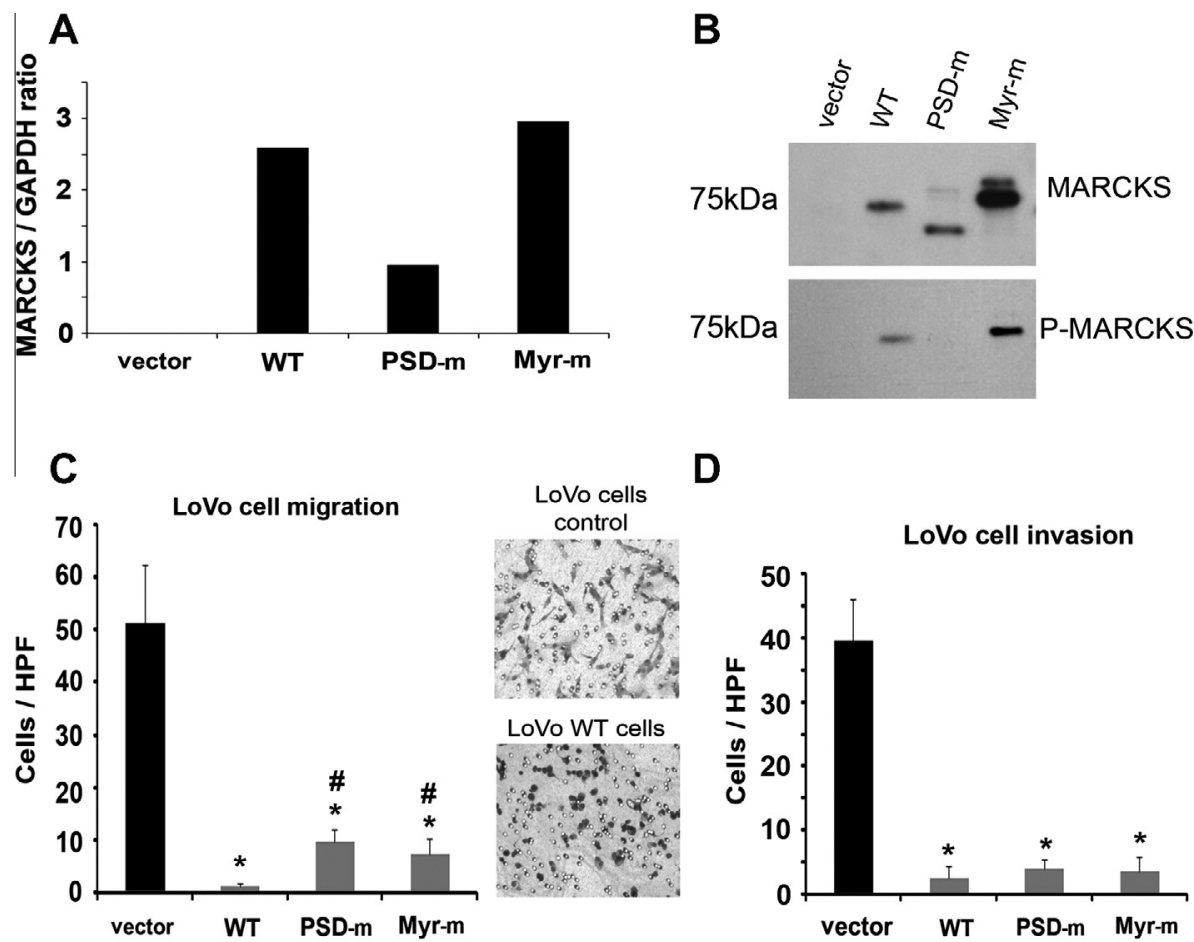

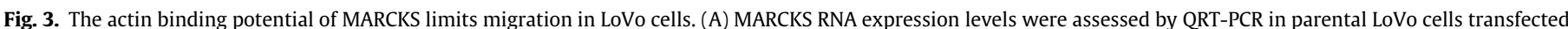

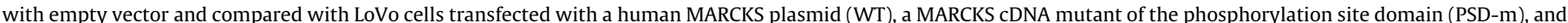



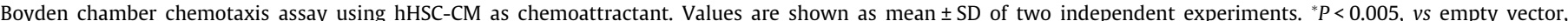

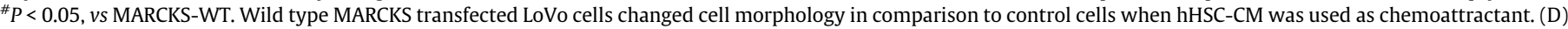
Invasion Matrigel assay was performed using hHSC-CM as chemoattractant. Values are shown as mean \pm SD of two independent experiments, ${ }^{*} P<0.05, v s$ empty vector. 
3.3. The actin binding potential of MARCKS limits migration of LoVo cells

Next we investigated whether cell motility was affected by reexpressing MARCKS in LoVo cells which do not normally express MARCKS protein. LoVo cells were transfected with a wild type (WT) MARCKS plasmid and two different MARCKS cDNA harbouring mutations i.e. a myristoylated mutant (Myr-m) and a deleted mutant of the PSD (PSD-m). As shown in Fig. 3A and B, MARCKS mRNA and protein expression were detectable in all three different cDNA transfected cellular conditions but not in parental empty vector transfected cells. In addition, phosphorylated MARCKS was detectable in WT and Myr-m transfected cells, whereas the PSD$\mathrm{m}$ transfected cells were negative for phosphorylated MARCKS, confirming that the phosphorylation site domain of MARCKS is mutated (Fig. 3B).

Migration of LoVo cells transfected with WT MARCKS and mutated variants revealed a remarkable reduction in cell motility (Fig. 3A). These findings indicate that the re-expression of MARCKS in LoVo cells affects the actin cytoskeleton reorganization during migration and that both the phosphorylation and the myristoylation of MARCKS are of equal importance for MARCKS proper cycling between the cytosol and the plasma membrane in order to regulate cell motility. Interestingly, LoVo cells that migrated towards hHSC-CM changed morphology when MARCKS expression was re-expressed. Empty vector-transfected cells showed mesenchymal-like features whereas LoVo cells transient transfected with MARCKS cDNA WT featured an epithelial phenotype (Fig. 3C). The re-expression of MARCKS and cDNA mutants resulted in an even higher inhibitory effect on the invasive capacity of LoVo cells. Empty vector transfected control cells invaded the Matrigel-coated filter, whereas all transfected LoVo cells did not invade (Fig. 3D). Overall, these data demonstrate that MARCKS acts as a key regulator of cell motility.

\subsection{MARCKS affects AURKB expression in Clone A cells and LoVo cells}

In a previous study we demonstrated that MARCKS is important for proper mitosis and that RNAi against MARCKS results in a prolonged cell cycle and AURKB delocalization [17]. In this study the effect of changing MARCKS protein expression on proliferation was analysed. Moreover, a possible link between MARCKS and AURKB in Clone A and LoVo cells was investigated.

RNAi against MARCKS expression in Clone A cells and reexpression in LoVo cells inhibited cell proliferation (Fig. 4A). The inhibitory effect of changing MARCKS expression on cell proliferation coincided with a down-regulation of AURKB protein expression in both cells lines as shown in Fig. 4B. Next, in two independent experiments, Clone A and LoVo cells were silenced against AURKB and western blot analysis showed that MARCKS protein expression was not affected when Clone A cells were silenced against AURKB (Fig. 4C). This finding suggests that an actin filament binding protein such as MARCKS can affect AURKB protein synthesis, but AURKB does not reciprocally affect MARCKS protein expression.
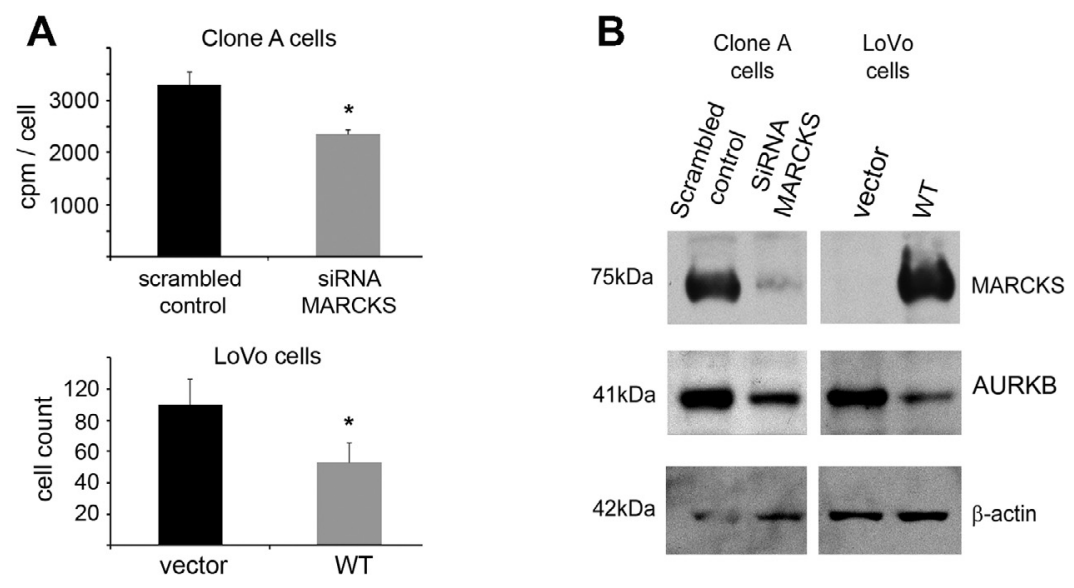

C
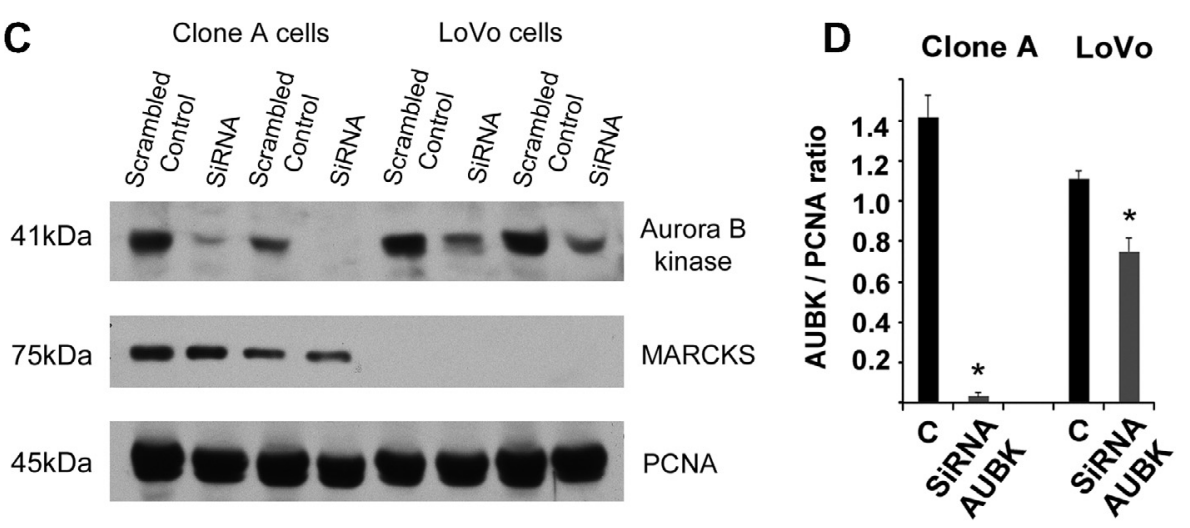

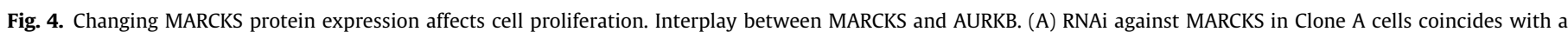

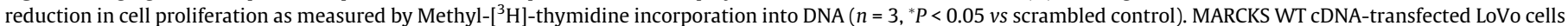

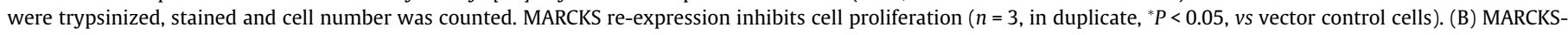

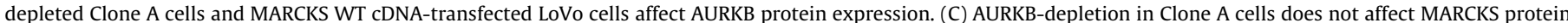

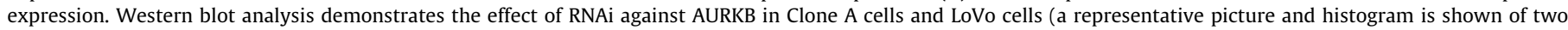
independent experiments, ${ }^{*} P<0.005$ ). 
3.5. Down-regulation of MARCKS in CT26 cells inhibits migration and invasion in vitro

The effect of MARCKS-depletion was assessed in the murine CT26 colon carcinoma cell line in a group of in vitro experiments instrumental for setting an in vivo model of liver metastasization. As shown in Fig. 5A, CT26 cells were stable shRNA transfected against MARCKS and cell migration and invasion were investigated. Stable shRNA MARCKS-depleted cells showed a decrease in cell motility in comparison to shRNA scrambled control cells towards mHSC-CM (Fig. 5B). As shown in Fig. 5C, cellular invasion of shRNA-treated MARCKS cells was reduced in comparison to scrambled control cells when mHSC-CM was used as chemoattractant.

\subsection{MARCKS depletion delays duration time of mitosis in CT26 cells}

Previous experimental evidence provided by our group suggested a key role of MARCKS in regulating the actin cytoskeleton during cell cycle in primary hHSC [17]. In this set of experiments the different phases of cell division of MARCKS-depleted CT26 cells and shRNA scrambled control cells were monitored by obliqueillumination video time lapse-imaging and this to investigate the role of MARCKS during mitosis in neoplastic cells such as CT26 cells. More specifically, the duration time of mitosis (DTM) of each mitotic cell was measured. Stably MARCKS-depleted CT26 cells showed a clear difference in phenotype during interphase in comparison to the scrambled shRNA control cells. These cells showed the typical epithelial phenotype whereas shRNA MARCKS-depleted cells showed a mesenchymal-like phenotype (Fig. 6A). ShRNAMARCKS-depleted cells were inhibited in proliferation when measured by employing MTT assay (Fig. 6B). Next, scrambled control cells showed an average DTM of $160 \mathrm{~min}(n=54$, Fig. 6C and Supplementary Video 6.1), whereas mitotic shRNA MARCKS-depleted cells are characterized by a significant increase in DTM when compared to scrambled control cells ( $n=43$, Fig. 6D and Supplementary Video 6.2 ). These data demonstrate that more than $51.2 \%$ of this cell population is characterized by a marked increase in DTM when compared with the average DTM of scrambled control cells (Fig. 6E). This feature coincided with an aberrant formation of the cleavage furrow followed by a delay in cleavage furrow ingression and pinching-off of the two daughter cells (Supplementary Video 6.2 and 6.3). In parallel, MARCKS protein expression was analysed by Western blot analysis (Fig. 6F).

\subsection{Down-regulation of endogenous MARCKS in CT26 cells antagonizes the metastatic ability in vivo}

A CT26 murine colorectal liver metastasis assay, syngeneic to $\mathrm{BALB} / \mathrm{c}$ mice, was performed in vivo to compare tumour formation between CT26 scrambled control cells and MARCKS-shRNA treated cells. Stable shRNA-transfected CT26 cells were used in these experiments and western blot analysis showed a strong reduction in MARCKS protein expression as shown in Fig. 7A. When mice were tumour-challenged, the suppression of MARCKS protein significantly decreased metastatic burden compared to the CT26tumour challenged mice (Fig. 7B). Liver metastasis in CT26 tumour-challenged mice demonstrated to contain 5-15 multiple macroscopic nodules occurring in a single liver (Fig. 7C). In contrast, few or no metastases were found macroscopically on the surface of the liver of those mice that were tumour-challenged with CT26 shRNA-depleted of MARCKS (Fig. 7B). This was confirmed by histology examination of performing haematoxylineosin stained sections (Fig. 7D). In contrast to the liver, tumour formation was found at the primary site of injection, i.e. spleen, in both conditions under investigation (Fig. 7B). These data confirm the previously observed inhibitory effect of MARCKS depletion on cell migration and invasion in vitro.

\section{Discussions}

MARCKS, a major PKC substrate, has been shown to be involved in the tumourigenesis of pituitary and thyroid tumours, breast cancer, cholangiocarcinoma and in glioblastoma multiforme. Alterations in the PKC-MARCKS-actin axis have been shown to affect processes such as motility, proliferation, invasion, adhesion, cell spreading and membrane trafficking [3,7,8,29]. Moreover, recent studies have identified MARCKS as a frequent target of mutation in colorectal carcinomas and small intestinal adenocarcinomas with MSI-H [9-11]. Overall the fine molecular mechanisms through which MARCKS affects tumour progression during CRC development are largely unknown.

The reorganization of the actin cytoskeleton is defined as the primary mechanism of cell motion during migration and cell invasion that is orchestrated by actin filament binding proteins such as
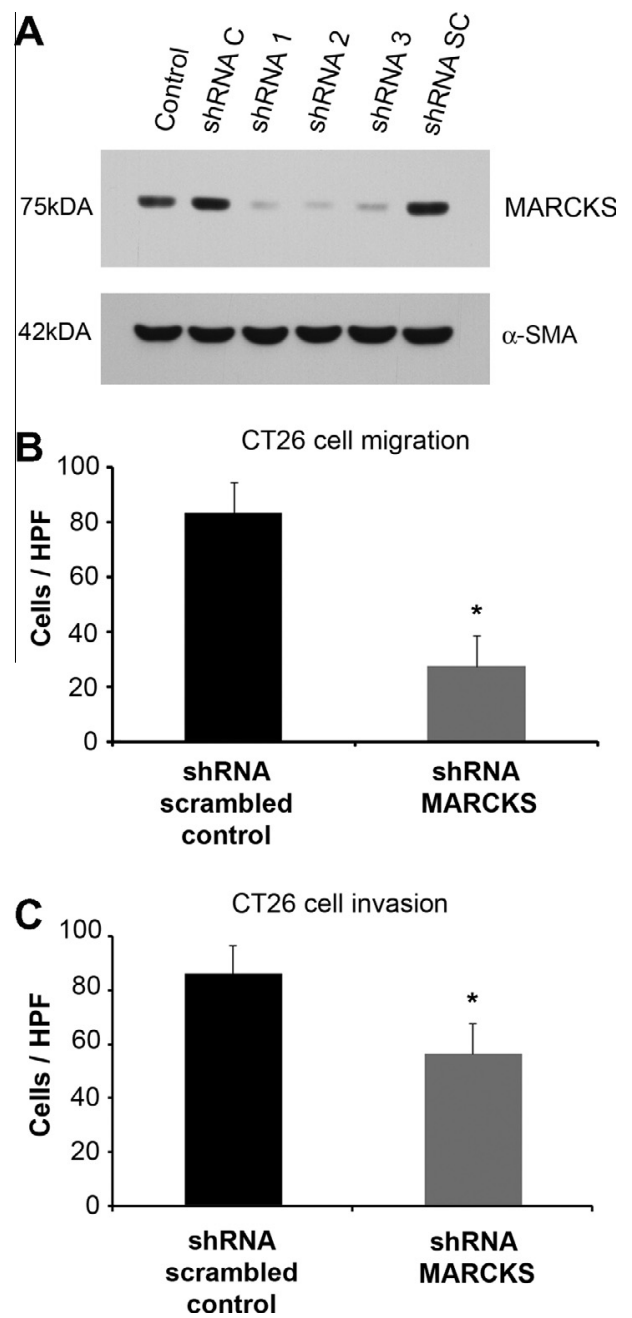

Fig. 5. Down-regulation of MARCKS expression inhibits migration and invasion of CT26 in vitro. (A) Western blot analysis showed the transfection efficiency of CT26 cells stable shRNA-depleted for MARCKS with different shRNA MARCKS (constructs 1-3), shRNA-C is empty vector and shRNA-SC is the scrambled control. (B) A Boyden chamber chemotaxis assay was used with $\mathrm{mHSC}-\mathrm{CM}$ as chemoattractant. Values are shown as mean $\pm \mathrm{SD}$ of three independent experiments. ${ }^{*} P<0.005$ vs shRNA scrambled control. (C) Invasion Matrigel assays were performed using mHSC-CM as chemoattractant. Values are shown as mean \pm SD of three independent experiments. Invasion was reduced when $\mathrm{MHSC}-\mathrm{CM}$ was used as chemoattractant. ${ }^{*} P<0.005$ vs shRNA scrambled control cells. 

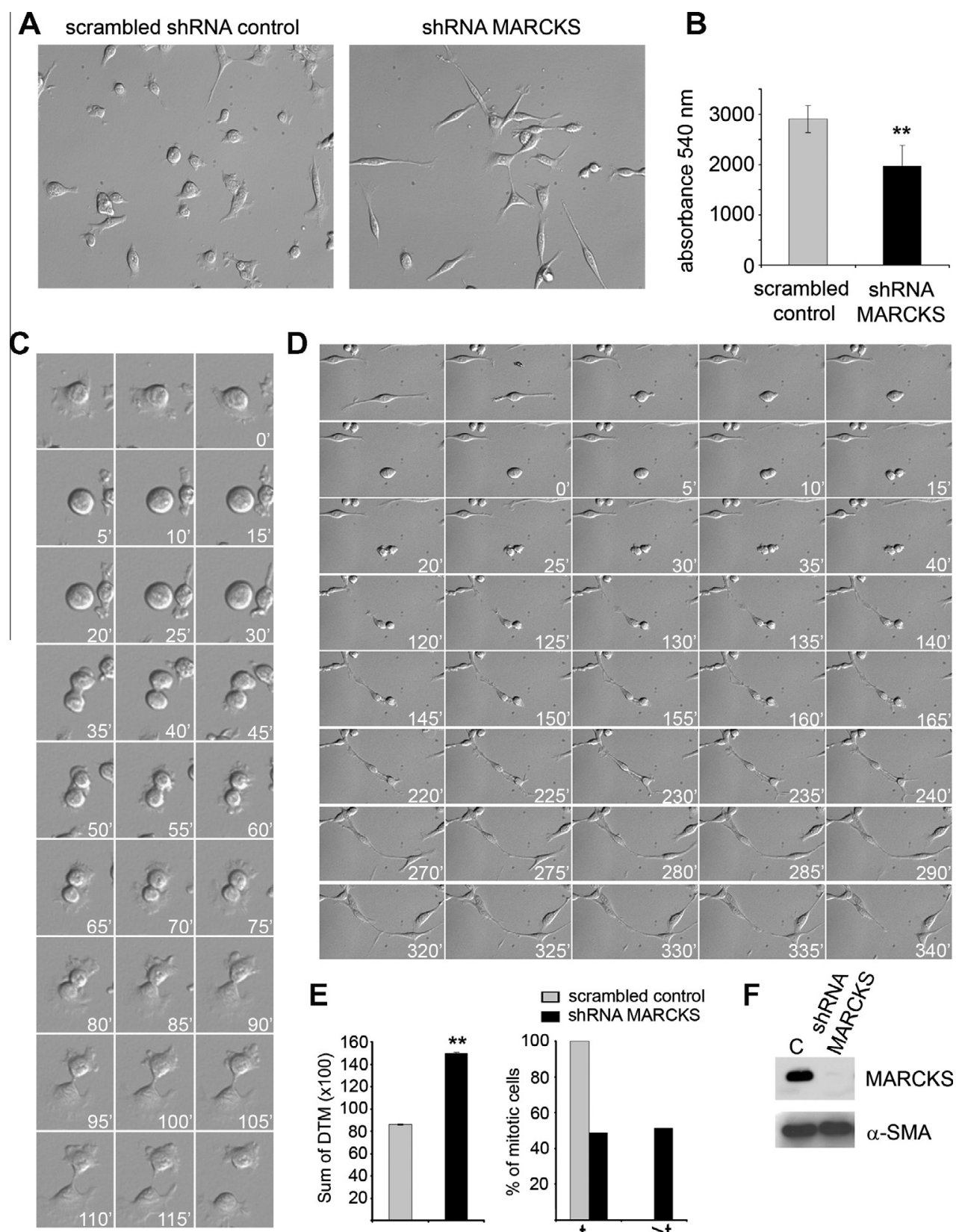

D
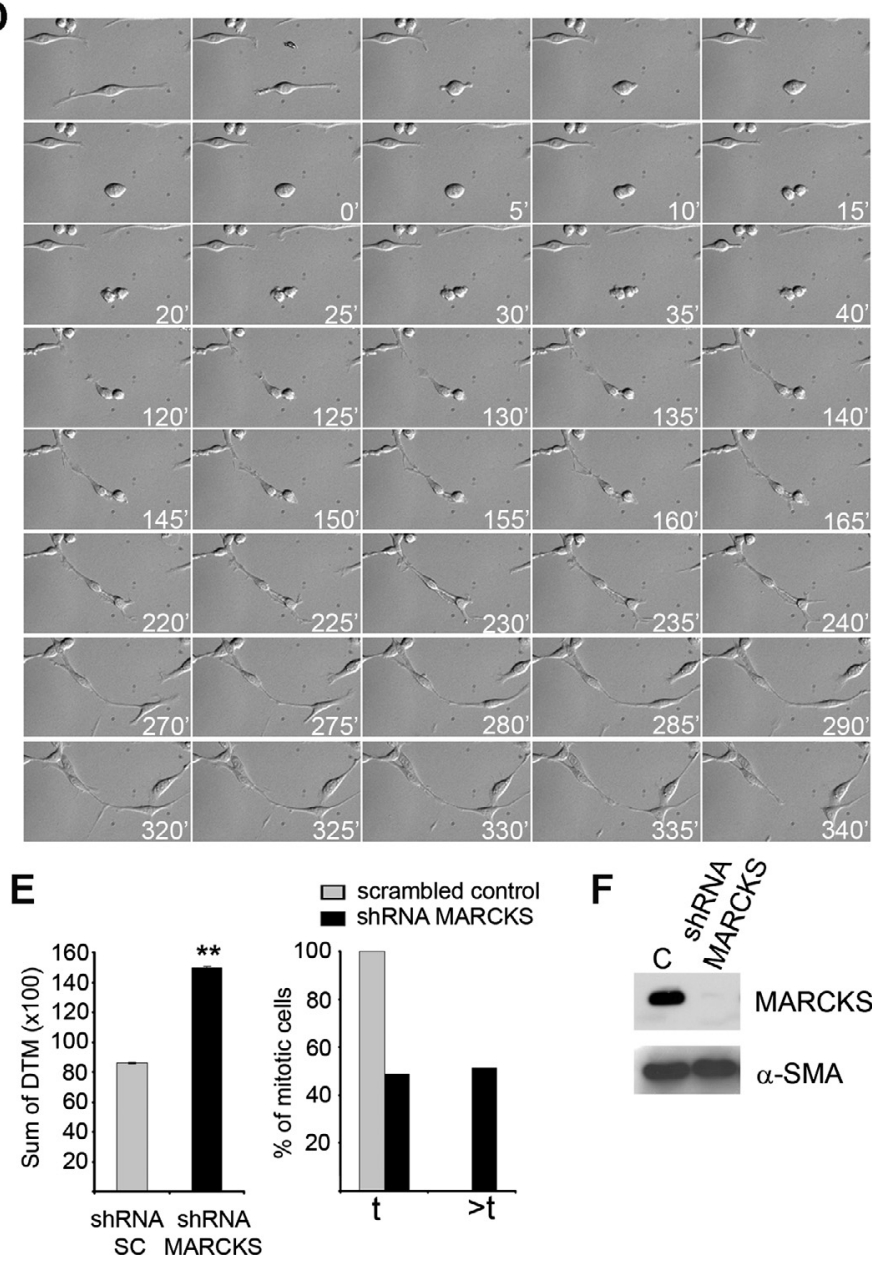

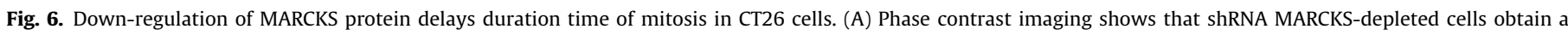

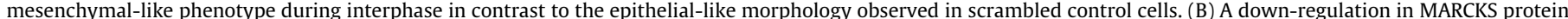

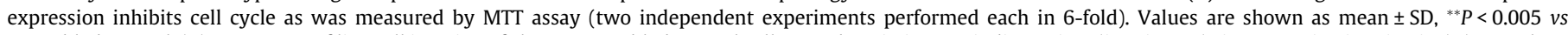

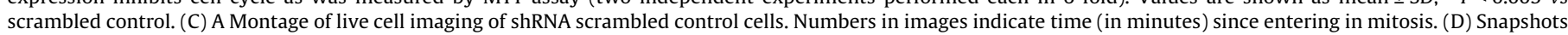





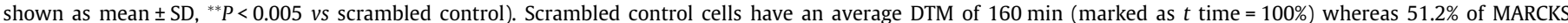

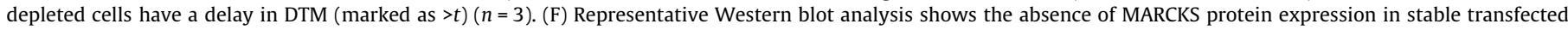
shRNA MARCKS-depleted cells ( $n=$ at least 3 ).

MARCKS [1,2]. In a previous study we have demonstrated the key role of MARCKS in the modulation of platelet-derived growth factor (PDGF)-induced hHSC motility [23]. These cells are liver-specific multifunctional mesenchymal cells known to play a key role in human liver pathophysiology [30-32], including a distinct stromal contribution to the development to primary and metastatic liver cancers [20-22]. In the present investigation we demonstrate that colon cancer cell motility and invasion in response to an established stromal extracellular stimulus, i.e. HSC-CM, are strongly affected by modulating MARCKS expression. Indeed, by altering intracellular MARCKS expression and therefore actin cytoskeleton dynamics, the effect of HSC-CM was abolished in both human and murine colorectal carcinoma cell lines. These changes are related to modifications of the fine-tuned balance between actin and MARCKS regulating reversible and local protein-membrane interactions ensuring cell plasticity, polarity and surface charge [33-35] in response to extracellular stimuli. Exogenous overexpression of MARCKS and mutational gain-on experiments revealed that MARCKS is an important determinant of cell motility and that the myristoylated domain and the phosphorylation site 

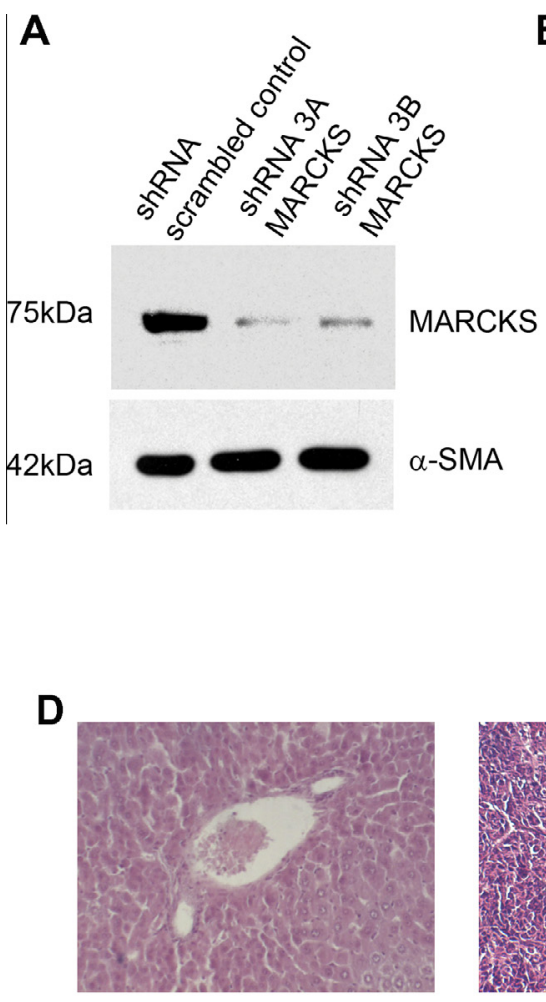

SHAM
B
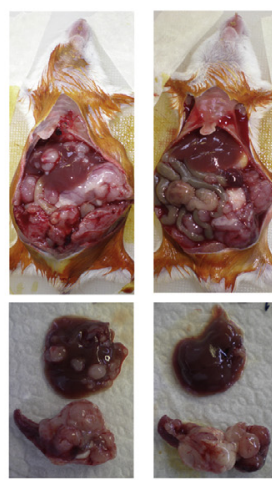

shRNA

$S C$
C
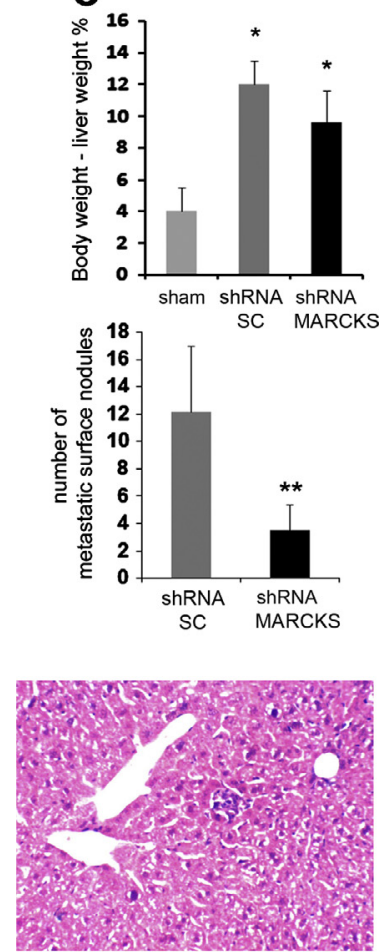

shRNA MARCKS

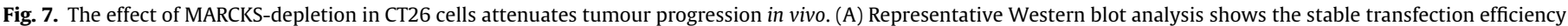

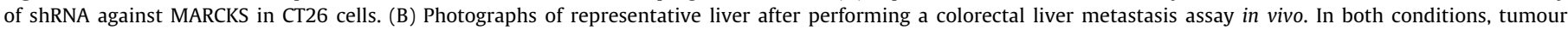

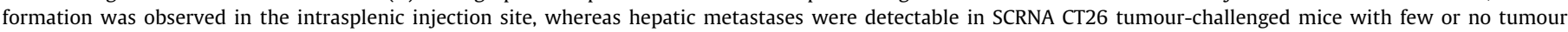

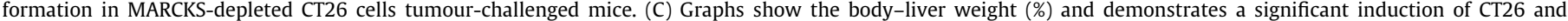



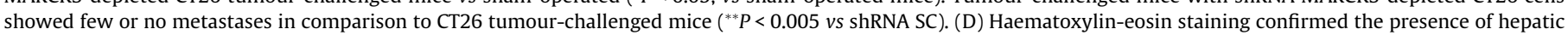
tumours in CT26 tumour-challenged mice.

domain of MARCKS are of equal importance to re-organize the membrane actin cytoskeleton during migration.

In addition to colon cancer cell motility and invasion, MARCKS expression may have also relevant implication on the cell cycle, as suggested by several studies [5,36,37]. Since mitosis is characterized by a dynamic remodelling of the cortical actin network $[38,39]$, the relationship between MARCKS, a key factor in actin reorganization, and AURBK, an important key mitotic checkpoint $[15,40]$, was further investigated. Changes in MARCKS protein expression, and thus the actin cytoskeleton, led to modifications in AURBK expression as shown by gain-on and gain-off experiments in both Clone A cells and LoVo cells. These data suggest that the re-organization of the actin cytoskeleton has a major impact on mitotic regulators such as AURKB, in agreement with recent data from our group [17].

The relationship between MARCKS expression and colon cancer cell biology was further confirmed in CT26 murine colon carcinoma cells in experiments preparatory to the establishment of the syngeneic in vivo model. Depletion of MARCKS resulted in reduced cell motion and prolonged cell cycle time suggesting a possible inhibitory effect on tumour growth at the metastatic site. Indeed, mice tumour-challenged with control cells developed a larger number of liver metastases when compared with mice tumourchallenged with MARCKS-depleted cells thus confirming that MARCKS affects tumour progression in vivo. Altogether, the in vitro and in vivo observations obtained by MARCKS-depletion in CT26, may recapitulate the clinical-pathological characteristics of MSI-H CRC, a CRC subtype which is characterized by unique molecular features and has distinct and relatively unknown mech- anisms by which colorectal cancer arise [41]. MARCKS activity has been shown to be impaired in MSI-H CRC as a consequence of a frameshift mutation and, although MSI-H CRC are poorly differentiated, they tend to be associated with a better patient survival prognosis [42] also in reason of a significantly lower rate of distant metastases [43]. An additional or alternative MARCKS-related mechanism explaining the reduced incidence of metastasis in this type of CRC is the immune reaction elicited by the secretion of a frameshift-mutated protein consequent to the mutation of MARCKS and recognized as an antigen characterized by a high immunogenic potential $[9,44,45]$.

Taken together, the results of the present study delineate a complex and articulated role of MARCKS in the progression of colorectal cancer. MARCKS supports colon cancer cell motility, invasion and proliferation whereas lack of MARCKS markedly affects these cancer features and reduces metastasization. Consequently, MARCKS might represent a suitable target to interfere and overcome the invasive behaviour of colon carcinoma cells at primary and distant sites.

\section{Acknowledgments}

This work was supported by Grant 2009.1294 from "Ente Cassa di Risparmio di Firenze" (A.G.) and in part by "Associazione Italiana per la Ricerca sul Cancro" (AIRC), "Istituto Toscano Tumori” (ITT), the University of Florence and "Ricerca Regionale in materia di salute “D.D. n. 3242 del 30/06/09” (M.P.).

The authors declare that they have no competing financial interests. 


\section{Appendix A. Supplementary material}

Supplementary data associated with this article can be found, in the online version, at http://dx.doi.org/10.1016/j.canlet.2013.01. 040 .

\section{References}

[1] H. Yamaguchi, J. Condeelis, Regulation of the actin cytoskeleton in cancer cell migration and invasion, Biochim. Biophys. Acta 1773 (2007) 642-652.

[2] C.G. Dos Remedios, D. Chhabra, M. Kekic, I.V. Dedova, M. Tsubakihara, D.A Berry, N.J. Nosworthy, Actin binding proteins: regulation of cytoskeletal microfilaments, Physiol. Rev. 83 (2003) 433-473.

[3] Y. Zhu, Q. Dong, B.J. Tan, W.G. Lim, S. Zhou, W. Duan, The PKCalpha-D294G mutant found in pituitary and thyroid tumors fails to transduce extracellular signals, Cancer Res. 65 (2005) 4520-4524.

[4] C.S. Jin, M. La, J.K. Ahn, G.G. Meadows, C.O. Joe, Tob-mediated cross-talk between MARCKS phosphorylation and ErbB-2 activation, Biochem. Biophys. Res. Commun. 283 (2001) 273-277.

[5] S. Manenti, F. Malecaze, H. Chap, J.M. Darbon, Overexpression of the myristoylated alanine-rich $C$ kinase substrate in human choroidal melanoma cells affects cell proliferation, Cancer Res. 58 (1998) 1429-1434.

[6] J.S. Jarboe, J.C. Anderson, C.W. Duarte, T. Mehta, S. Nowsheen, P.H. Hicks, A.C. Whitley, T.D. Rohrbach, R.O. McCubrey, S. Chiu, T.M. Burleson, J.A. Bonner, G.Y. Gillespie, E.S. Yang, C.D. Willey, MARCKS regulates growth and radiation sensitivity and is a novel prognostic factor for glioma, Clin. Cancer Res. 18 (2012) 3030-3041.

[7] J. Micallef, M. Taccone, J. Mukherjee, S. Croul, J. Busby, M.F. Moran, A. Guha, Epidermal growth factor receptor variant III-induced glioma invasion is mediated through myristoylated alanine-rich protein kinase $\mathrm{C}$ substrate overexpression, Cancer Res. 69 (2009) 7548-7556.

[8] A. Techasen, W. Loilome, N. Namwat, E. Takahashi, E. Sugihara, A. Puapairoj, M. Miwa, H. Saya, P. Yongvanit, Myristoylated alanine-rich C kinase substrate phosphorylation promotes cholangiocarcinoma cell migration and metastasis via the protein kinase C-dependent pathway, Cancer Sci. 101 (2010) 658-665.

[9] N.G. Kim, H. Rhee, L.S. Li, H. Kim, J.S. Lee, J.H. Kim, N.K. Kim, H. Kim, Identification of MARCKS, FLJ11383 and TAF1B as putative novel target genes in colorectal carcinomas with microsatellite instability, Oncogene 21 (2002) 5081-5087.

[10] T. Yamaguchi, T. Iijima, T. Mori, K. Takahashi, H. Matsumoto, H. Miyamoto, T. Hishima, M. Miyaki, Accumulation profile of frameshift mutations during development and progression of colorectal cancer from patients with hereditary nonpolyposis colorectal cancer, Dis. Colon Rectum 49 (2006) 399-406.

[11] S. Michel, M. Kloor, S. Singh, G. Gdynia, W. Roth, D.M. von Knebel, P. Schirmacher, H. Blaker, Coding microsatellite instability analysis in microsatellite unstable small intestinal adenocarcinomas identifies MARCKS as a common target of inactivation, Mol. Carcinogenesis 49 (2010) 175-182.

[12] J.H. Hartwig, M. Thelen, A. Rosen, P.A. Janmey, A.C. Nairn, A. Aderem, MARCKS is an actin filament crosslinking protein regulated by protein kinase $C$ and calcium-calmodulin, Nature 356 (1992) 618-622

[13] F. Wohnsland, A.A. Schmitz, M.O. Steinmetz, U. Aebi, G. Vergeres, Interaction between actin and the effector peptide of MARCKS-related protein. Identification of functional amino acid segments, J. Biol. Chem. 275 (2000) 20873-20879.

[14] M. Tatsuka, H. Katayama, T. Ota, T. Tanaka, S. Odashima, F. Suzuki, Y. Terada, Multinuclearity and increased ploidy caused by overexpression of the auroraand Ipl1-like midbody-associated protein mitotic kinase in human cancer cells, Cancer Res. 58 (1998) 4811-4816.

[15] J.R. Bischoff, L. Anderson, Y. Zhu, K. Mossie, L. Ng, B. Souza, B. Schryver, P. Flanagan, F. Clairvoyant, C. Ginther, C.S. Chan, M. Novotny, D.J. Slamon, G.D. Plowman, A homologue of Drosophila aurora kinase is oncogenic and amplified in human colorectal cancers, EMBO J. 17 (1998) 3052-3065.

[16] S. Ruchaud, M. Carmena, W.C. Earnshaw, The chromosomal passenger complex: one for all and all for one, Cell 131 (2007) 230-231.

[17] K. Rombouts, T. Mello, F. Liotta, A. Galli, A. Caligiuri, F. Annunziato, M. Pinzani, MARCKS actin-binding capacity mediates actin filament assembly during mitosis in human hepatic stellate cells, Am. J. Physiol. Cell Physiol. 303 (2012) C357-C367.

[18] M. Pinzani, P. Failli, C. Ruocco, A. Casini, S. Milani, E. Baldi, A. Giotti, P. Gentilini, Fat-storing cells as liver-specific pericytes. Spatial dynamics of agoniststimulated intracellular calcium transients, J. Clin. Invest. 90 (1992) 642-646.

[19] D. Cassiman, T. Roskams, Beauty is in the eye of the beholder: emerging concepts and pitfalls in hepatic stellate cell research, J. Hepatol. 37 (2002) 527-535.

[20] A. Mazzocca, R. Coppari, F.R. De, J.Y. Cho, T.A. Libermann, M. Pinzani, A. Toker, A secreted form of ADAM9 promotes carcinoma invasion through tumorstromal interactions, Cancer Res. 65 (2005) 4728-4738.
[21] Z.F. Yang, R.T. Poon, J. To, D.W. Ho, S.T. Fan, The potential role of hypoxia inducible factor 1 alpha in tumor progression after hypoxia and chemotherapy in hepatocellular carcinoma, Cancer Res. 64 (2004) 5496-5503.

[22] C. Coulouarn, A. Corlu, D. Glaise, I. Guenon, S.S. Thorgeirsson, B. Clement, Hepatocyte-stellate cell cross-talk in the liver engenders a permissive inflammatory microenvironment that drives progression in hepatocellular carcinoma, Cancer Res. 72 (2012) 2533-2542.

[23] K. Rombouts, B. Lottini, A. Caligiuri, F. Liotta, T. Mello, V. Carloni, F. Marra, M. Pinzani, MARCKS is a downstream effector in platelet-derived growth factorinduced cell motility in activated human hepatic stellate cells, Exp. Cell Res. 314 (2008) 1444-1454.

[24] G.M. Verghese, J.D. Johnson, C. Vasulka, D.M. Haupt, D.J. Stumpo, P.J. Blackshear, Protein kinase C-mediated phosphorylation and calmodulin binding of recombinant myristoylated alanine-rich C kinase substrate (MARCKS) and MARCKS-related protein, J. Biol. Chem. 269 (1994) 9361-9367.

[25] M. Pinzani, L. Gesualdo, G.M. Sabbah, H.E. Abboud, Effects of platelet-derived growth factor and other polypeptide mitogens on DNA synthesis and growth of cultured rat liver fat-storing cells, J. Clin. Invest. 84 (1989) 1786-1793.

[26] B.K. Carney, S. Caruso, V.L. Cassimeris, The microtubule cytoskeleton is required for a G2 cell cycle delay in cancer cells lacking stathmin and p53, Cytoskeleton (Hoboken) 69 (2012) 278-289.

[27] X. Zhu, J. Wang, K. Moriguchi, L.T. Liow, S. Ahmed, I. Kaverina, M. Murata-Hori, Proper regulation of Cdc42 activity is required for tight actin concentration at the equator during cytokinesis in adherent mammalian cells, Exp. Cell Res. 317 (2011) 2384-2389.

[28] S.R. Johnstone, A.K. Best, C.S. Wright, B.E. Isakson, R.J. Errington, P.E. Martin Enhanced connexin 43 expression delays intra-mitotic duration and cell cycle traverse independently of gap junction channel function, J. Cell Biochem. 110 (2010) 772-782.

[29] T. Bai, S.W. Luoh, GRB-7 facilitates HER-2/Neu-mediated signal transduction and tumor formation, Carcinogenesis 29 (2008) 473-479.

[30] S.L. Friedman, Hepatic stellate cells: protean, multifunctional, and enigmatic cells of the liver, Physiol. Rev. 88 (2008) 125-172.

[31] M. Pinzani, J. Macias-Barragan, Update on the pathophysiology of liver fibrosis, Expert. Rev. Gastroenterol. Hepatol. 4 (2010) 459-472.

[32] V. Hernandez-Gea, S.L. Friedman, Pathogenesis of liver fibrosis, Annu. Rev. Pathol. 6 (2011) 425-456.

[33] N.M. Goldenberg, B.E. Steinberg, Surface charge: a key determinant of protein localization and function, Cancer Res. 70 (2010) 1277-1280.

[34] S. McLaughlin, A. Aderem, The myristoyl-electrostatic switch: a modulator of reversible protein-membrane interactions, Trends Biochem. Sci. 20 (1995) $272-276$.

[35] T. Laux, K. Fukami, M. Thelen, T. Golub, D. Frey, P. Caroni, GAP43, MARCKS, and CAP23 modulate $\mathrm{PI}(4,5) \mathrm{P}(2)$ at plasmalemmal rafts, and regulate cell cortex actin dynamics through a common mechanism, J. Cell Biol. 149 (2000) 14551472.

[36] T. Herget, S.F. Brooks, S. Broad, E. Rozengurt, Expression of the major protein kinase $C$ substrate, the acidic 80-kilodalton myristoylated alanine-rich $C$ kinase substrate, increases sharply when Swiss 3T3 cells move out of cycle and enter G0, Proc. Natl. Acad. Sci. USA 90 (1993) 2945-2949.

[37] J.C. Reed, U. Rapp, M.P. Cuddy, Transformed 3T3 cells have reduced levels and altered subcellular distribution of the major PKC substrate protein MARCKS, Cell Signal. 3 (1991) 569-576.

[38] E. Boucrot, T. Kirchhausen, Mammalian cells change volume during mitosis, PLoS One 3 (2008) e1477.

[39] A. Carvalho, A. Desai, K. Oegema, Structural memory in the contractile ring makes the duration of cytokinesis independent of cell size, Cell 137 (2009) 926-937.

[40] P. Steigemann, C. Wurzenberger, M.H. Schmitz, M. Held, J. Guizetti, S. Maar, D.W. Gerlich, Aurora B-mediated abscission checkpoint protects against tetraploidization, Cell 136 (2009) 473-484.

[41] C.C. Pritchard, W.M. Grady, Colorectal cancer molecular biology moves into clinical practice, Gut 60 (2011) 116-129.

[42] M. Guidoboni, R. Gafa, A. Viel, C. Doglioni, A. Russo, A. Santini, T.L. Del, E. Macri, G. Lanza, M. Boiocchi, R. Dolcetti, Microsatellite instability and high content of activated cytotoxic lymphocytes identify colon cancer patients with a favorable prognosis, Am. J. Pathol. 159 (2001) 297-304.

[43] A. Buckowitz, H.P. Knaebel, A. Benner, H. Blaker, J. Gebert, P. Kienle, D.M. von Knebel, M. Kloor, Microsatellite instability in colorectal cancer is associated with local lymphocyte infiltration and low frequency of distant metastases, $\mathrm{Br}$. J. Cancer 92 (2005) 1746-1753.

[44] K.T. You, L.S. Li, N.G. Kim, H.J. Kang, K.H. Koh, Y.J. Chwae, K.M. Kim, Y.K. Kim, S.M. Park, S.K. Jang, H. Kim, Selective translational repression of truncated proteins from frameshift mutation-derived mRNAs in tumors, PLoS Biol. 5 (2007) e109.

[45] F.M. Speetjens, M.M. Lauwen, K.L. Franken, C.M. Janssen-van Rhijn, D.S. van S.A. Bres, C.J. van de Velde, C.J. Melief, P.J. Kuppen, S.H. van der Burg, H. Morreau, R. Offringa, Prediction of the immunogenic potential of frameshiftmutated antigens in microsatellite instable cancer, Int. J. Cancer 123 (2008) 838-845. 